NASA Technical Memorandum 84506 NASA-TM-84506 19820026315

\title{
STIMULATION OF THE FLUCTUATING FIELD OF A FORCED JET
}

Alvin Bayliss, Lucio Maestrello, and Eli Turkel

AUGUST 1982 
- 


\section{SIMULATION OF THE FLUCTUATING FIELD OF A FORCED JET}

Alvin Bayliss

Courant Institute of Mathematical Sciences

Luc1o Maestre110

NASA Langley Research Center

E11 Turke1

Te1. Aviv Unfversity

\section{Abstract}

The fluctuating field of a jet excited by transient mass injection is simulated numerically. The model is developed by expanding the state vector as a mean state plus a fluctuating state. Nonlinear terms are not neglected and the effect of nonlinearity is studied. The results show a significant spectral broadening in the flow field due to the nonlinearity. In addition, large scale structures are broken down into smaller scales.

*This work was partlally supported by NASA Contracts No. NAS1-14472 and NAS116394 for the first and third authors were in residence at ICASE, NASA Langley Research Center, Hampton, VA. Additional support for the first author was provided by the U. S. Air Force Contract No. AFOSR-76-2881 and U. S. Department of Energy Contract No. DE-AC02-76ER03077. 
Introduction

A numerical method for simulating the axisymmetric fluctuating field of a forced circular jet has been developed. The method is based on a solution of the Euler equations for homentropic flow in cylindrical coordinates. The jet is forced by transient point mass injection. The source strength is scaled by a parameter $\varepsilon$ and the state vector $W=(\bar{p}, \bar{p}, \bar{u}, \bar{v})$ is expanded as a mean state plus a fluctuating state of order $\varepsilon$. Here $p$ is the pressure, $\rho$ the density and $u$ and $v$ are the axial and radial velocity components respectively.

The solution is expanded in the form $\bar{p}=p_{0}+\varepsilon p$, etc. where the mean state (with subscript " 0 ") is assumed to satisfy the unforced Euler equations. The variables $(p, p, u, v)$ will represent the fluctuating field in response to the jet forcing. New variables $e=\left(\rho_{0}+\varepsilon \rho\right) u$ and

$f=\left(\rho_{0}+\varepsilon \rho\right) \mathbf{v}$ are introduced for simplicity. With cylindrical spatial coordinates $z$ (axial) and $r$ (radial) the Euler equations for the fluctuating field become

$$
\begin{aligned}
& \rho_{t}+\left(\rho U_{0}+\rho_{0} u\right)_{z}+\left(\rho v_{0}+\rho_{0} v\right)_{r}+\frac{\left(\rho v_{0}+\rho_{0} v\right)}{r}=m \\
& e_{t}+\left(e\left(U_{0}+\varepsilon u\right)\right)_{z}+\left(e\left(V_{0}+\varepsilon v\right)\right)_{r}+p_{z}+f U_{0 r}-e v_{0 r}-\frac{\varepsilon f u}{r}=\varepsilon m u \\
& f_{t}+\left(f\left(U_{0}+\varepsilon u\right)\right)_{z}+\left(f\left(V_{0}+\varepsilon v\right)\right)_{r}+p_{r}+e U_{0 z}-f v_{0 z}-\frac{\varepsilon f v}{r}=\varepsilon m v
\end{aligned}
$$

In the development of system (1) it is assumed that the derivatives of the mean pressure and density in space can be neglected. It is an exact consequence of the full Euler equations with a source of mass injection, and of the expansion into mean and fluctuating states. The source is $\varepsilon \mathrm{m}$ (units density/time). The mean velocities $U_{0}$ and $V_{0}$ are taken from measurements of a spreading jet. The system is solved in a cylindrical rectangle including a semi-infinite pipe from which the jet exits. The computational domain is illustrated in Fig. 1 .

The use of the quasi-momentum variables $e$ and $f$ gives a system from which the fluctuating field can be computed directly rather than as a small part of the total field. In addition the linear limit can be recovered by simply setting $\varepsilon=0$ in (1). The nonlinear terms are explicitly exhibited. The fluctuating pressure $p$ is obtained from the density $\rho$ by the homentropic relation

$$
\overline{\mathrm{p}}=\overrightarrow{A \rho}=\mathrm{P}_{0}\left(1+\varepsilon \frac{\rho}{\rho_{0}}\right)^{\gamma}
$$

where $\gamma=1.4$ in air.

The fundamental assumption entering into the derivation of (1) is that the mean state is a solution to the unforced Euler equations. This is not true for a state determined from experimental measurements. Numerical experiments have verified however, that the qualitative features of the fluctuating solution are insensitive to small changes in the mean state and thus we belleve that the solution to (1) qualitatively represents the fluctuating field in response to a a given source.

The source is assumed to be a delta function in space (modelled by a sharp Gaussian) with a pulse-like time dependence. The source location, $z_{s}$, 
Is on the jet centerline approximately 1.2 jet diameters downstream of the nozzle exit. Specifically

where the function $f(t)$ is

$$
\underset{\text { is }}{m(t, z, r)}=f(t) \delta\left(r^{2}+\left(z-z_{s}\right)^{2}\right),
$$

$$
f(t)=e^{-\left(a t^{2}+b t^{-2}\right)},
$$

for suitable constants $a$ and $b$. The use of (4) permits the investigation of a broad band spectrum.

The fluctuating field described by (1) reduces to the acoustic field for large distances. The near fleld and flow fleld are dominated by instability waves which are generated because the mean flow is linearly unstable. The pulse is assumed to dominate the natural sources of jet noise. These natural sources are both the linear and nonlinear terms in (1). In real jet these terms are determined from the turbulent fluctuations whereas in the numerical model these natural sources are excited by the pulse. The important physical effect is the generation of packets of instability waves in the flow. Large scale structures which are related to mean flow instablifties have been observed experimentally in both forced and unforced jets $[1,2]$. These structures interact with and modify the resulting acoustic fields. The model permits this interaction to be studied in both the Iinear $(\varepsilon=0)$ and nonlinear $(\varepsilon \neq 0)$ regimes. Calculations with the linear model and comparison with experiments are described elsewhere $[3,4]$. In the rest of this paper we describe the numerical requirements in order to compute with this model and the effects of the nonlinear terms on the fluctuating fleld.

\section{Numerical Model}

In order to numerically integrate (1) it is necessary to resolve the solution over large length scales (far field, near fleld, and flow field). This necessitates the use of a higher order difference scheme. The system can be written in the form

$$
\mathrm{w}_{\mathrm{t}}+\mathrm{F}_{\mathrm{z}}+\mathrm{G}_{\mathrm{r}}=\mathrm{H}
$$

where $w$ is the vector $(\rho, e, f)$ and $F, G$, and $H$ are appropriate functions. Equation (5) is split into two one-dimensional operators in $z$ and $r$. Each 1-d system is integrated by using a fourth order version of the MacCormack scheme [5]

$$
\begin{aligned}
& \widetilde{w}_{1}^{n+1}=w_{1}^{n}+\frac{\Delta t}{6 \Delta x}\left(-7 F_{1}+8 F_{1+1}-F_{1-2}\right)+\Delta t H_{1} \\
& w_{1}^{n+1}=1 / 2\left(\widetilde{w}_{1}^{n+1}+w_{1}^{n}+\frac{\Delta t}{6 \Delta x}\left(7 \widetilde{F}_{1}-8 \widetilde{F}_{1-1}+\widetilde{F}_{1-2}\right)+\Delta t \widetilde{H}_{1}\right),
\end{aligned}
$$

together with a symmetric variant $\left(\mathrm{H}_{1}\right.$ is obtained from some splitting of H). Typlcal grids require of the order of 40,000 grid points over distances of the order of 50 jet diameters in all directions. Our experfence has been that on problems of this size, second order schemes are not sufficient to obtain accurate solutions with the amount of resolution that is feastble. The explicit scheme (6) naturally lends itself to vectorization and has been implemented on the CDC CYBER-203 with great efficiencies. 
In addition it is necessary to Impose boundary conditions which accurately simulate outgoing radiation at the far field boundaries. A family of radiation boundary conditions has been developed whtch provide increasingly accurate approximations to outgoing radiation. The leading member of this family is

$$
p_{t}+\rho_{\infty} c_{\infty} \tilde{u}_{t}+\frac{p}{d}=0,
$$

where $2^{c_{\infty}}$ fs the ambient sound speed and $\rho_{\infty}$ the ambient density. Here $\mathrm{d}^{2}=\mathrm{r}^{2}+\mathrm{z}^{2}$ and $\tilde{u}$ is the outgoing radial velocity based on a spherical coordinate system near the source m.

System (1) includes terms proportional to $r^{-1}$. This singularity at the axis is resolved by including these terms in the flux vector $G$ when $r=0$. In addition it is necessary to modify the difference formula (6) at boundarles. This is accomplished by introducing fictitious grid points outside of the computational domain and using a third order extrapolation of the flux function ( $F$ or $G$ ). This approach was found to be the most readily vectorizable. It has been verifled that the resulting scheme is fourth order accurate.

\section{Nonlinear Results}

We next describe results illustrating the effect of the nonlinear terms on the fluctuating field. In Figs. 2 and 3 the fluctuating pressure is shown as a function of axial location $z / D$ ( $D$ is the jet diameter) and non-dimensional time $t c_{\infty} / D$ for two different radial positions and for $\varepsilon=0.00$ and $\varepsilon=0.05$. All figures show an acoustic wave (speed of sound normalized to unity) in the downstream direction trailed by several much larger waves. These are Instablitty waves which travel with a speed of approximately $.7 U_{j}$ where $U_{f}=.66 c_{\infty}$ is the jet exit velocity. A series of acoustic ripples can also be seen propagating upstream. These are due to diffraction of the upstream acoustic wave by the nozzle lip.

The figures indicate that the nonlinear terms have little effect on the primary acoustic pulse and on the acoustic diffraction from the nozzle lip. The nonlinearity has a pronounced effect on the instability waves. Increasing the nonlinearity causes these predominantly large scale structures to break up into smaller scale structures. This can be seen in both the additional ripples which trail the instability waves and a sharpening of the individual pulses indicating an enhanced high frequency content. It is also evident that for increasing $\mathrm{r} / \mathrm{D}$ these smaller scale structures are comparable in amplitude to the primary instability waves.

In Figs. 4 and 5 the fluctuating vorticity is shown for two fixed times and for $\varepsilon=0.00$ and $\varepsilon=0.05$. The intense vortices in Fig. 4 correspond to the Instablifty waves In Figs. 2 and 3 while the vortices at the later time in Fig. 5 represent a residual shedding of vorticity from the nozzle lip. It is apparent from the figures that nonlinear effects tend to slow down the vortices as they propagate downstream. Thus the trailing vortices catch up with the leading vortices and a possible pairing of vortices can be observed. True vortex merging, which has been observed experimentally [6], depends heavily on viscosity as well as nonlinearity and is not simulated here. 
In Figs. 6a and $6 \mathrm{~b}$ the normalized power spectral densities (PSD) for the fluctuating axial velocity and pressure are plotted for the linear and nonlinear cases as a function of the Helmholtz number $f D / c_{\infty}$ (f is the frequency). These figures clearly indicate the shift into higher frequencies and the overall broadening of the spectra caused by the nonlinear effects. Then results are typlcal for the fluctuating fleld at all points.

\section{Discussion}

The fundamental difference between the nonlinear and Iinear computations is the breakdown of the large scale structures into smaller scale structures. This is assoctated with a transfer of energy into higher frequencles or equivalently a broadening of the spectral content of the fluctuating fleld. It is also illustrated by the increased interaction between the different vortices. In real jets this is a fundamental step in the transition to fully developed turbulence. The results indicate that at least the inftal stages of this energy cascade into smaller scales can be simulated just by the nonlinear terms of an axisymmetric and inviscid calculation.

The generation of smaller scale fluctuations fully justifles the use of fourth order spatial discretizations. The numerical scheme is accurate and is in general stable. Higher values of $\varepsilon$ can be readily computed although the Gaussian approximation to the $\delta$ function source will have to be smoothed out. 


\section{References}

[1] Crow, S. C. and Champagne, F. H.: Orderly Structure in Jet Turbulence, J. Fluid Mech., Vol. 48, Part 3, 1971, pp. 547-591.

[2] Maestre11o, L. and Fung, Y. T.: Quas1-Perlodic Structure of a Turbulent Jet, J. Sound \& Vib., Vo1. 64, 1979, pp. 107-122.

(31) Maestrello, L., Bayliss, A., and Turkel, E.: On the Interaction of a Sound Pulse with the Shear Layer of an Axisymmetric Jet, J. Sound \& Vib., Vol. 74, 1974, pp. 281-301.

[4] Bayliss, A. and Maestre1lo, L.: S1mulation of Instabilities and Sound Radiation in a Jet, AIAA J., Vol. 19, No. 7, 1981, pp. 835-841.

[5] Gottlieb, D. and Turkel, E.: Dissipative Two-Four Methods for Time-Dependent Problems, Math. Comput., Vol. 30, 1976, pp. 703-723.

[6] Ho, ChI-ming and Huang, Lein-saing: Subharmonics and Vortex Merging in Mixing Layers, to appear in J. Fluid. Mech., 1982. 


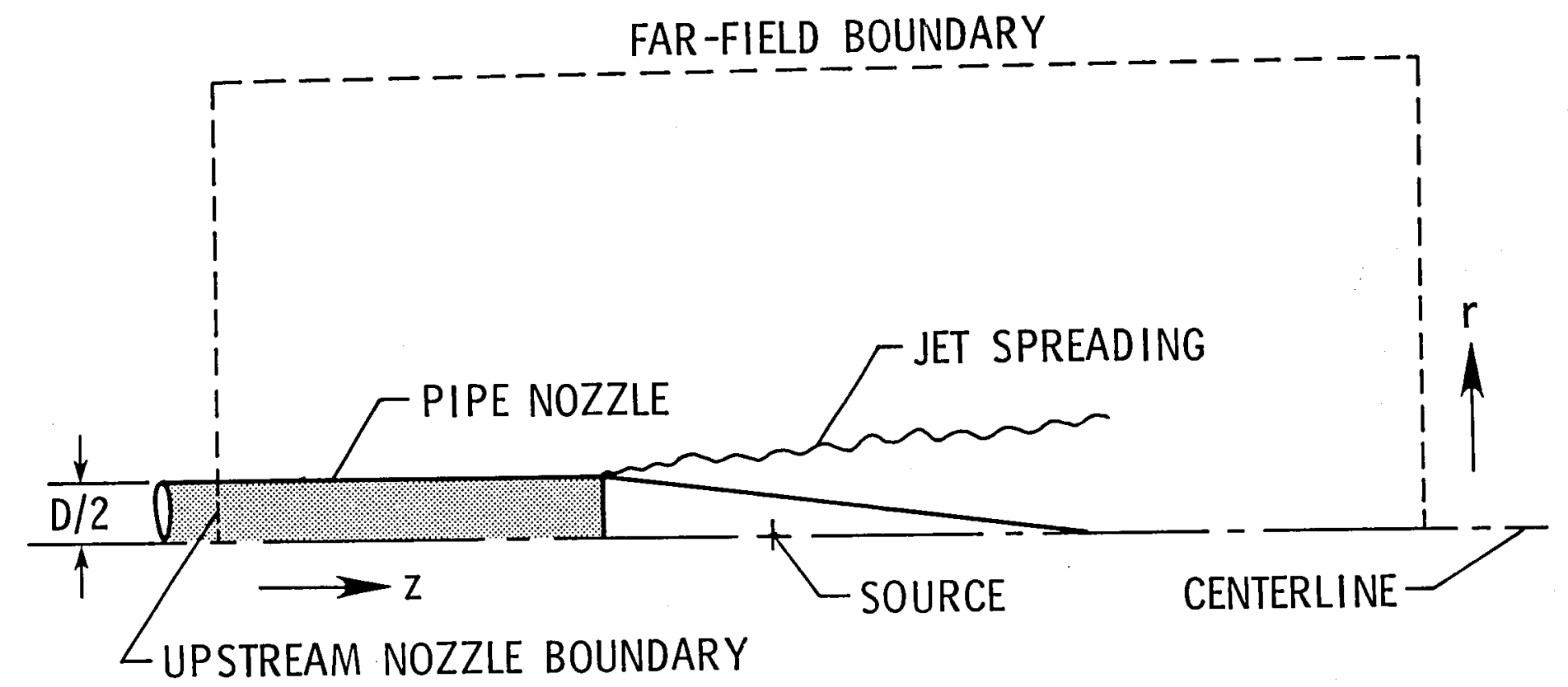

Figure 1. Computational Domain 

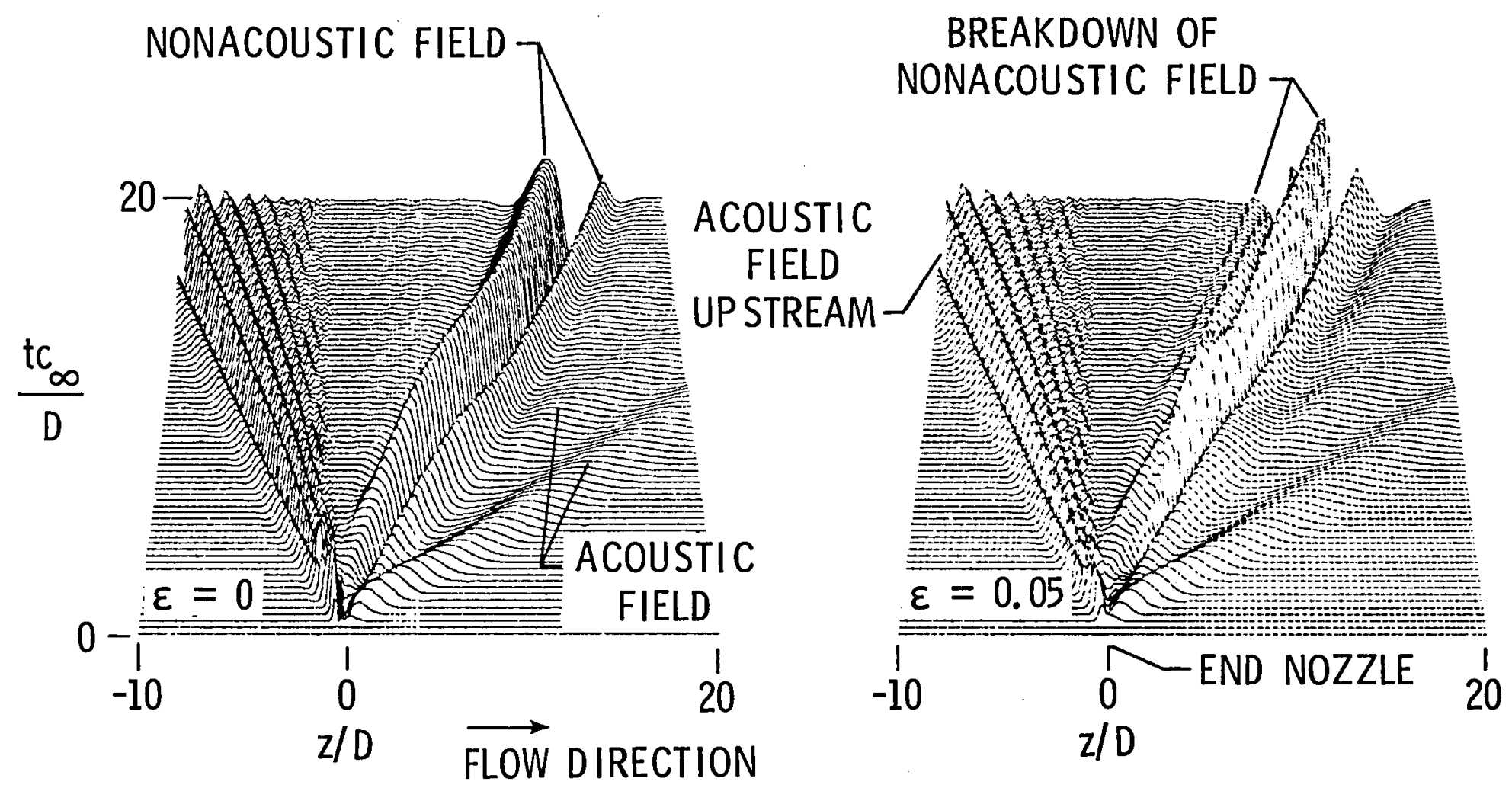

Figure 2. Three-dimensional plots of the fluctuating pressure $r / D=.29$. 


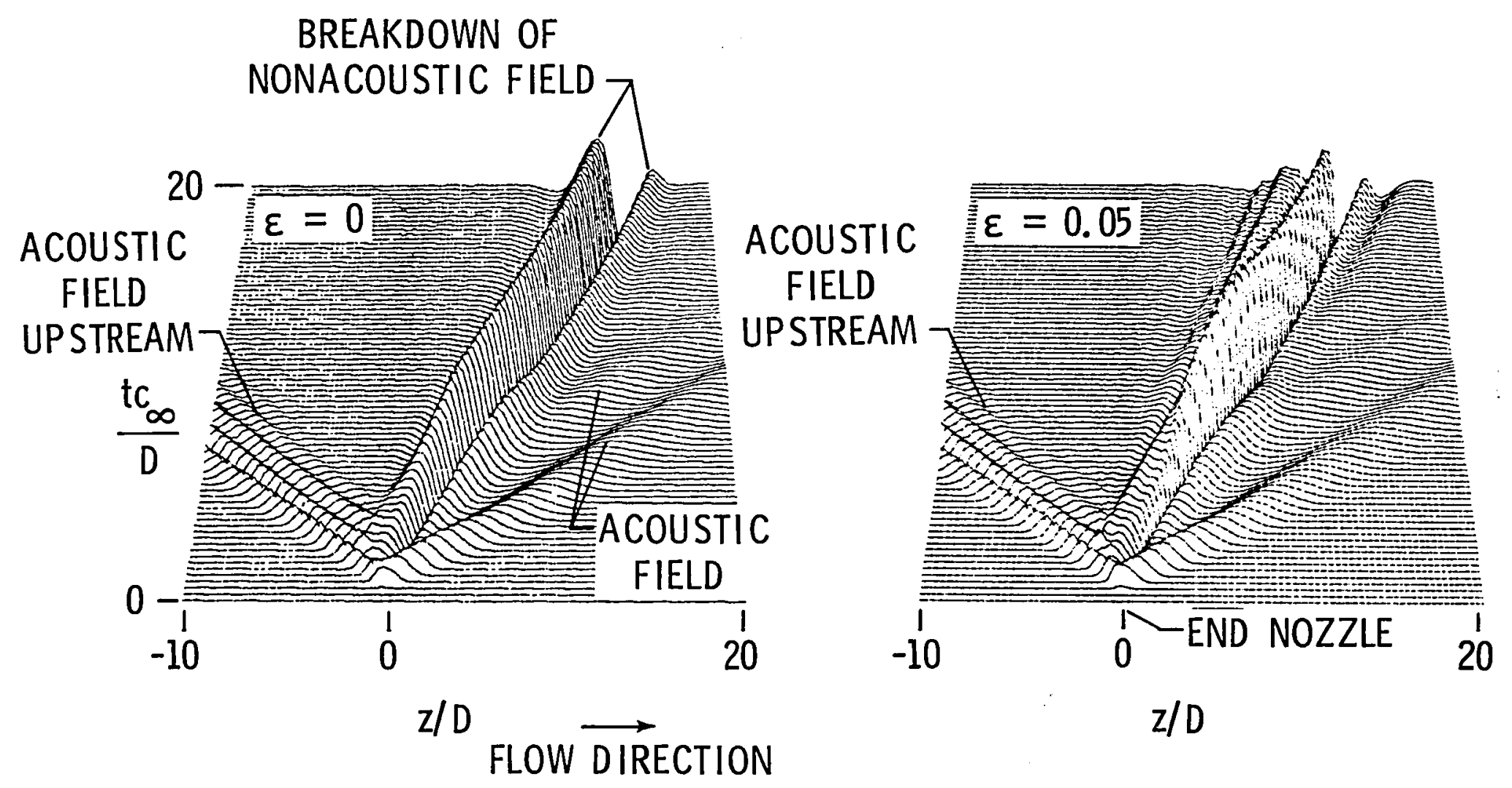

Figure 3. Three-dimensional plots of the fluctuating pressure $r / D=.61$. 


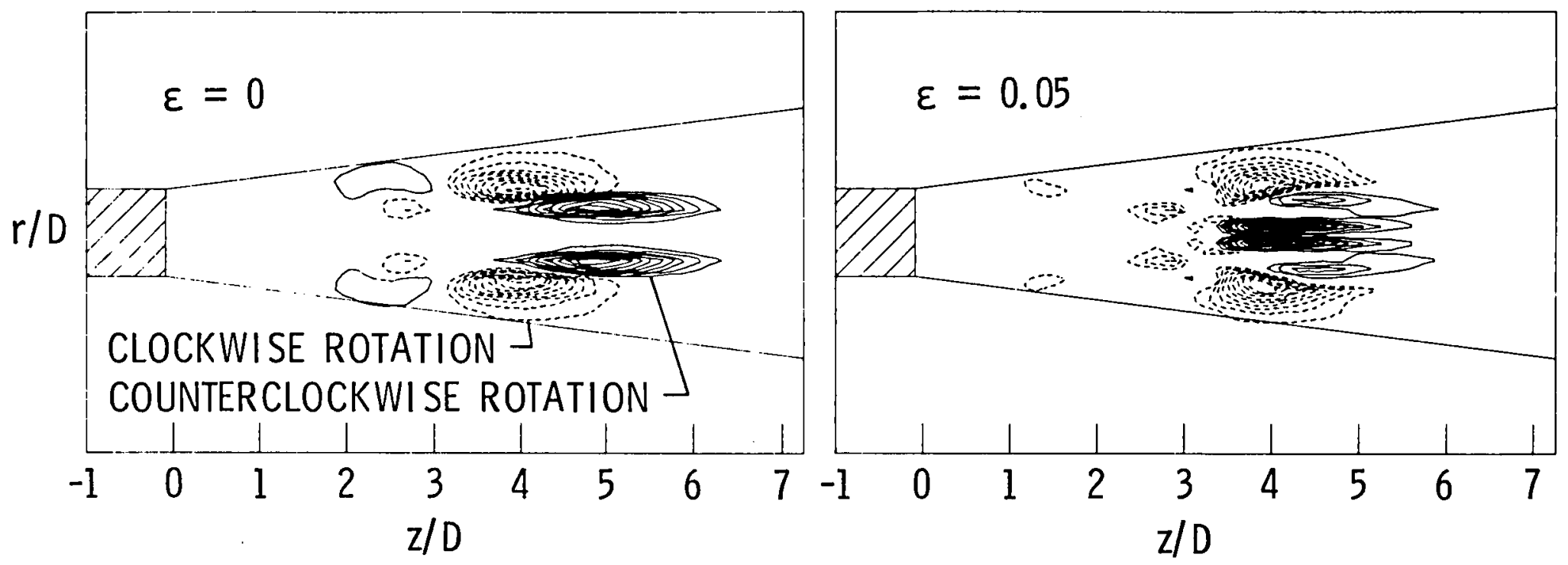

Figure 4. Fluctuating vorticity at $t c_{\infty} / D=10$. 


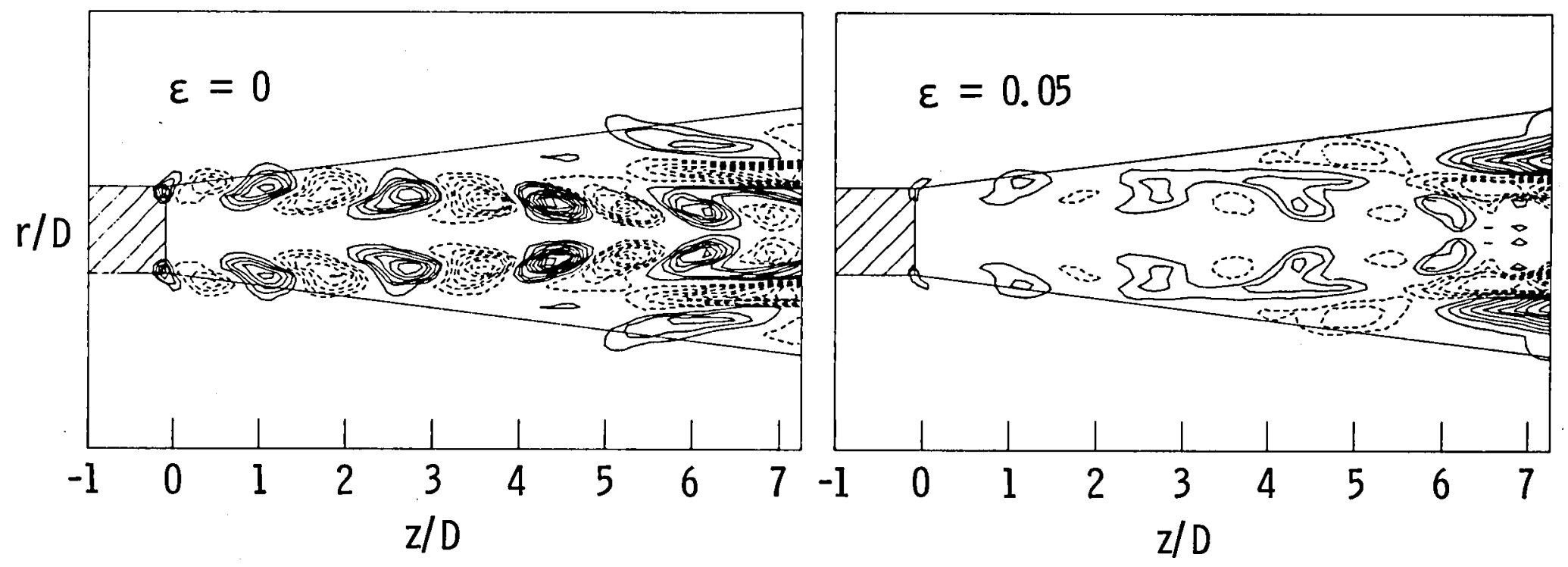

Figure 5. Fluctuating vorticity at $t c_{c o} / D=30$. 


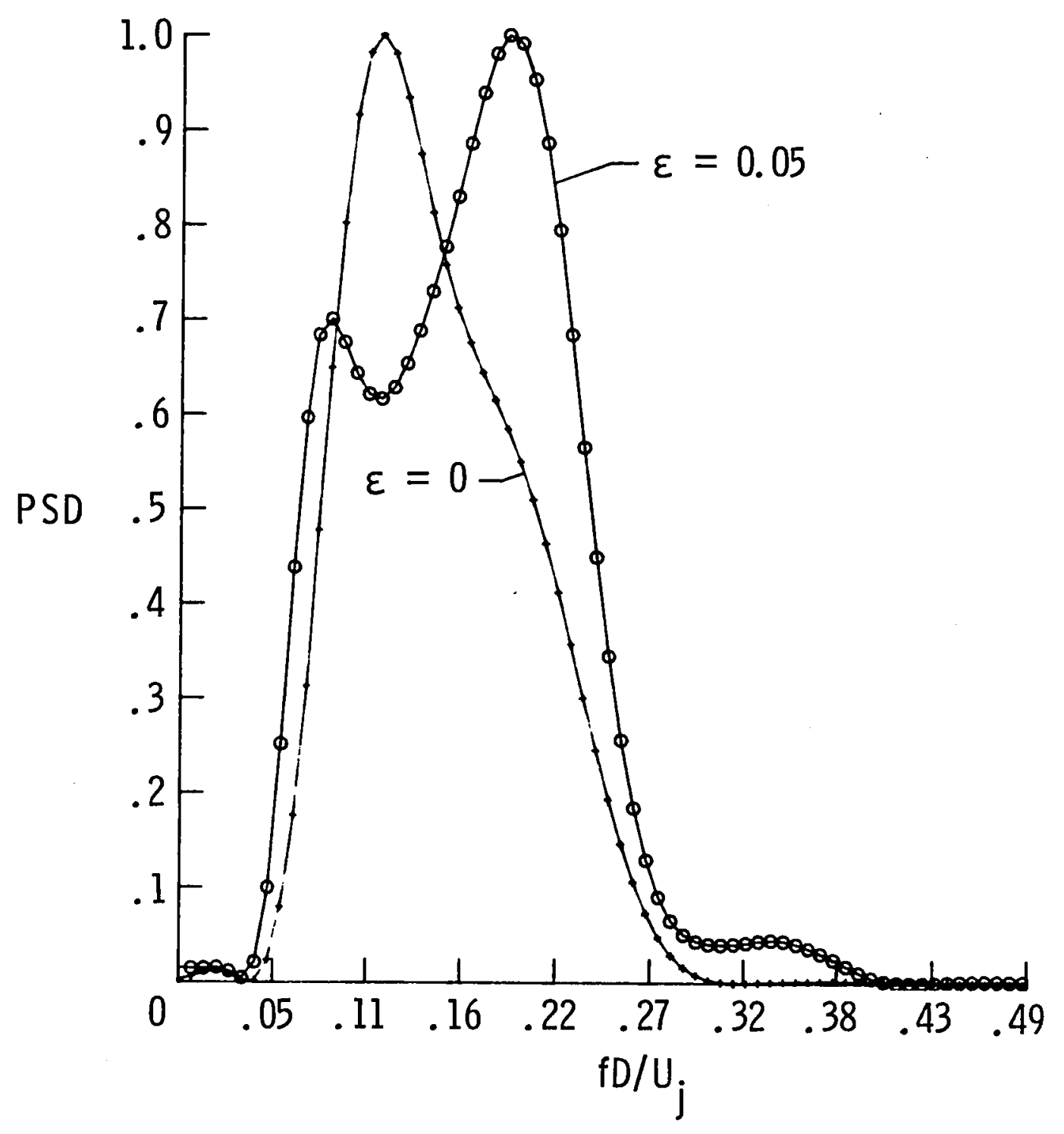

Figure 6a. Flow field velocity spectra $z / D=7.3, r / D=1.5$. 


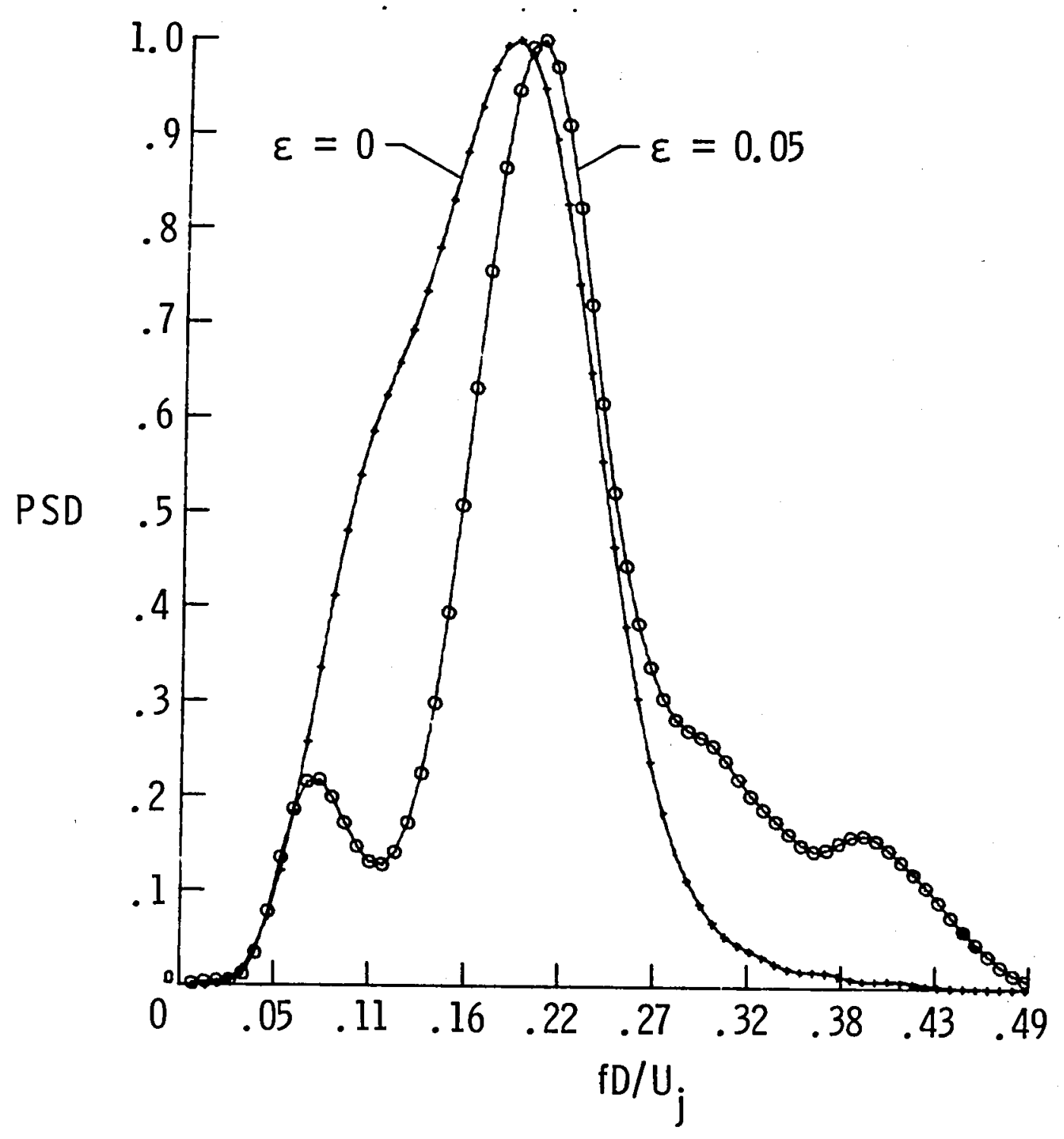

Figure 6b. Acoustic far-field spectra $z / D=38, r / D=20$. 


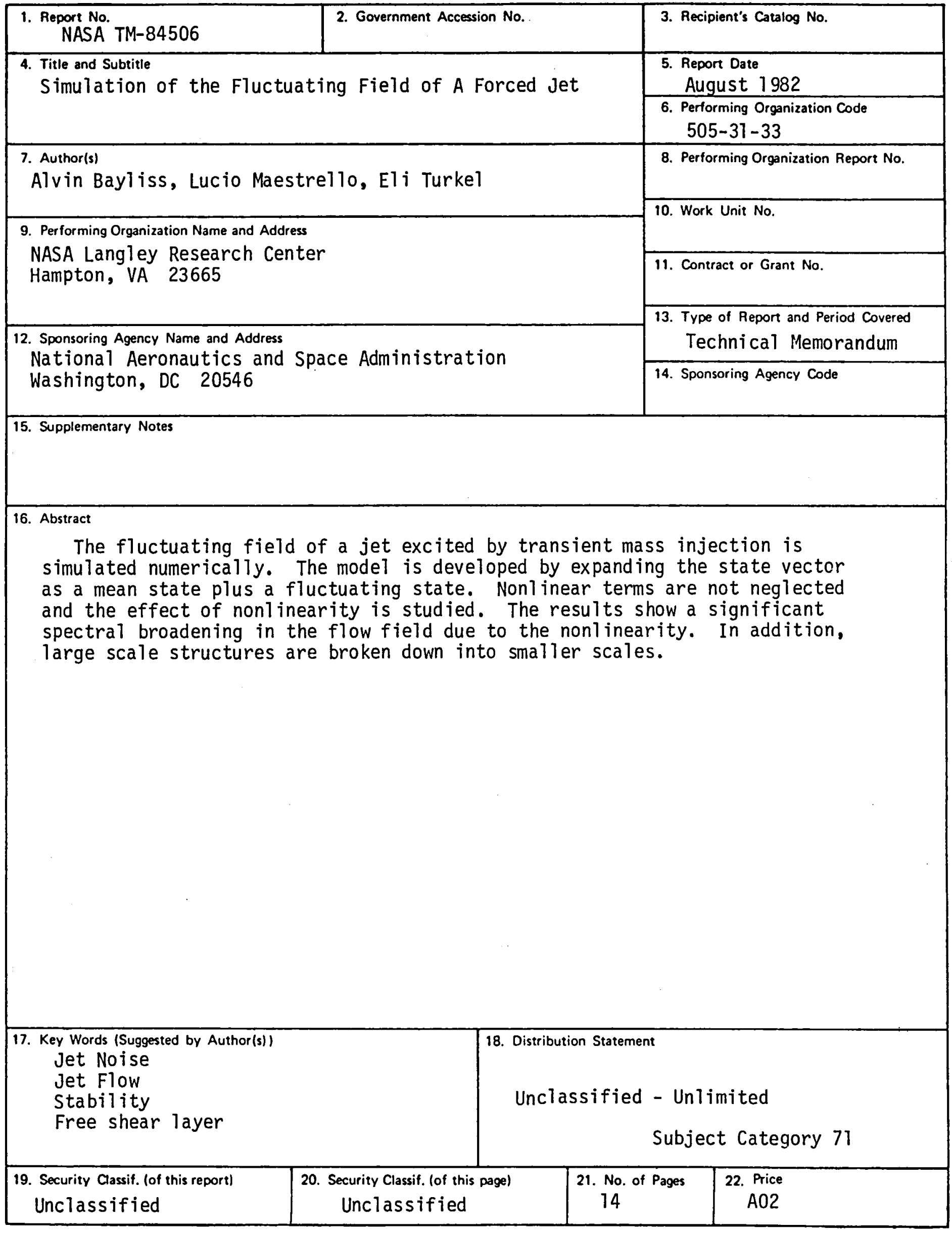


